\title{
El nuevo trabajo industrial: una mirada crítica desde la calidad del empleo ${ }^{1}$
}

\author{
The New Industrial Job: A Critical Analysis \\ from the Quality of the Job
}

\section{O novo trabalho industrial: um olhar crítico a partir da qualidade do emprego}

\author{
Martín Rodríguez Miglio ${ }^{2}$ \\ Profesor de la Universidad Nacional de General Sarmiento, Los Polvorines-Argentina \\ mrmiglio@ungs.edu.ar

\begin{abstract}
Analía Erbes ${ }^{3}$
Profesora de la Universidad Nacional de General Sarmiento, Los Polvorines-Argentina aerbes@ungs.edu.ar

\section{Sonia Roitter}

Profesora de la Universidad Nacional de General Sarmiento, Los Polvorines-Argentina sroitter@ungs.edu.ar
\end{abstract}

\section{Andrea Pujol ${ }^{5}$}

Profesora de la Universidad Nacional de Córdoba, Córdoba-Argentina

apujol@psyche.unc.edu.ar

Recibido: 01.02.14

Aprobado: 21.10 .14

1 Este trabajo se desarrolló en el marco del proyecto "Crecimiento, innovación y dinámica del empleo. Análisis de la industria argentina post-convertibilidad", financiado por la Agencia Nacional de Promoción Científica y Tecnológica (Ministerio de Ciencia, Tecnología e Innovación Productiva) y la Universidad Nacional de General Sarmiento.

2 Licenciado en Economía.

3 Magíster en Gestión de la Ciencia, la Tecnología y la Innovación.

4 Doctora en Ciencias Económicas, Mención en Economía.

5 Licenciada en Psicología. 


\title{
Resumen
}

En este trabajo se aborda la relación entre las nuevas formas de organización de la producción y la calidad del empleo, a partir de una revisión crítica de las perspectivas más tradicionales. En particular, se discute el impacto de la adopción de las nuevas formas de organización de la producción sobre la calidad del empleo. Para ello se analizan los cambios operados en el proceso de trabajo desde los inicios del capitalismo, especialmente los ocurridos en el último cuarto del siglo XX. Entre las principales conclusiones se destaca que los cambios producidos en los factores que configuran la organización productiva y la gestión del trabajo tienen un impacto discutible sobre la calidad del empleo y el bienestar de los trabajadores.

Palabras clave: Calidad de Empleo, Condiciones de Trabajo, Organización de la Producción, Organización del Trabajo, Postfordismo.

Clasificación JEL: L23; M54; J28; J81.

\begin{abstract}
In this article we address the relationship between new ways of organizing production and job quality. In doing this, we critically review the most traditional perspectives. Particularly, we examine the scope of the impact on job quality when adopting new ways of production structure. In doing this, changes made during the working process from the beginning of capitalism are analyzed, focusing on the changes that occurred during the last quarter of the 2oth Century. Some of the key findings point out that changes in the factors that shape productive organization and labor management have a questionable impact on job quality and workers' welfare.
\end{abstract}

Keywords: Job Quality, Working Conditions, Organization of Production, Work Organization, Postfordism.

\section{Resumo}

Este artigo discute a relação entre as novas formas de organização da produção e a qualidade do trabalho, a partir de uma revisão crítica de perspectivas tradicionais. Questionase em que medida a adoção destas novas formas de organização da produção impactaram a qualidade do trabalho. Para esta finalidade se analisa as mudanças acontecidas no processo de trabalho desde o início do capitalismo, particularmente aquelas que ocorreram no último quarto do século XX. Entre as principais conclusões pode-se destacar que as mudanças nos fatores que configuram a organização da produção e gestão do trabalho, têm um impacto discutível sobre a qualidade do emprego, do bem-estar, e do desenvolvimento dos trabalhadores.

Palavras-chave: Condições de Emprego, Qualidade de Trabalho, Organização da Produção, Organização do Trabalho, Pós-fordismo. 


\section{Introducción}

Existe cierto consenso en la literatura económica sobre el agotamiento del régimen de acumulación del tipo keynesiano-fordista que tuvo lugar en las estructuras productivas de los países centrales a mediados de los años setenta. Este escenario habría disparado una ofensiva del capital en pos de recomponer los niveles de productividad y, consecuentemente, los deprimidos niveles de la tasa ganancia. Como producto de esta tendencia, se observa una reconversión del aparato productivo que da lugar a nuevas formas de organizar el trabajo. Entre estas se destaca la introducción de flexibilidad técnica (en la composición y volumen de la producción) mediante la incorporación de la producción justo-atiempo y de la gestión de mano de obra (numérica, funcional, salarial), lo cual se traduce en la modificación de las estructuras organizativas (menos niveles jerárquicos, deslocalización y/o tercerización de actividades) y en la introducción de tecnologías sociales de gestión (trabajo en células, redes o equipos, círculos de calidad, grupos de mejora) bajo el fundamento de que favorecen una mayor implicación de los trabajadores (management participativo, management by stress).

Algunos autores aseguran que esta reconfiguración productiva habría impactado positivamente en la economía en su conjunto a partir de, entre otros factores, nuevas formas de cooperación entre empresarios y trabajadores que permitieron recomponer los niveles de productividad. Esta nueva relación se sustentaría en la creación de espacios dentro de la fábrica que harían posible la participación activa de los trabajadores y la alineación de objetivos orientados a incrementar el compromiso con la actividad laboral.

Por el contrario, algunas corrientes de pensamiento caracterizan a esta etapa como el inicio de la erosión de la relación salarial clásica, lo cual generó una reconfiguración de las relaciones laborales. Esto se tradujo en un marco de acción más individualizado y descolectivizado vía la fragilización de la acción colectiva o de la identidad colectiva (Bacon y Storey 1994; De la Garza, Gayosso y Moreno 2010), en una dilución de las diferencias de clase, en una "supertaylorización" y en la incorporación de una nueva dimensión en términos de explotación, vía absorción de las ideas. Todo esto impactaría en la calidad del empleo y de vida de los trabajadores (Vidal 2013). Por su parte, los aportes de las escuelas de sociología francesa cuestionan la situación actual por sus efectos en términos de sufrimiento en el trabajo (Dejours 2006), pérdida del lazo social y fragilización de identidades profesionales (Pérrilleux 2001; De Gaulejac 2005; Dubar 2002).

El presente trabajo se propone discutir en qué medida la adopción de las nuevas prácticas productivas impactan sobre la calidad del empleo, a partir de su contribución a la mejora de las condiciones de trabajo y al incremento de las posibilidades de desarrollo personal y profesional de los trabajadores operativos $^{6}$. Para ello, en la primera sección se revisan los cambios operados en los

6 El análisis realizado en este estudio se centra en la relación entre nuevas formas de organización de la producción y calidad del empleo, enfatizando fundamentalmente el impacto sobre los trabajadores operativos. Este recorte no implica desconocer que los cambios sucedidos en la organización del trabajo también impactaron fuertemente sobre los empleados de alta calificación, con importantes efectos sobre la calidad del empleo, tal como lo sostienen Loughlin y Murray (2013), entre otros. 
procesos de trabajo y en las formas de organización de la producción a partir de la década del setenta, considerando principalmente lo sucedido al interior de las firmas en el marco de este proceso de reconversión y su impacto sobre la dinámica productiva y laboral. En la segunda parte se estudian las condiciones laborales desde la perspectiva de la calidad del empleo que retoma, en particular, la importancia de la organización del trabajo como dimensión de análisis. A su vez, se analizan críticamente los distintos factores que dan cuenta de la organización productiva y la gestión del trabajo. En la tercera sección se plantean las reflexiones finales y principales conclusiones.

\section{Las nuevas formas de organización de la producción a partir de la década del setenta}

\subsection{Nuevas prácticas productivas: contexto y surgimiento}

El proceso de reconversión productivo, que comienza a consolidarse a partir de la década del setenta, estuvo acompañado por nuevas formas de organizar el trabajo, las cuales se cristalizaron, principalmente, en la implementación del trabajo en equipo y en la adopción de la producción justo a tiempo, aunque también se encuentra fuertemente asociado a múltiples prácticas organizativas tales como el sistema kan-ban, la autonomía del trabajador, la creciente participación y/o imbricación de los anteriores y el control de calidad descentralizado, entre otras cuestiones.

Estas prácticas generaron nuevas dinámicas de trabajo. En términos de Coriat (2001), una de las transformaciones fundamentales se relaciona con la línea de montaje (dispositivo más importante de la producción en masa), la cual permitió la parcelación y la repetitividad del trabajo y requirió una calificación obrera mínima y súper especializada. Los crecientes niveles de ausentismo, la alta rotación de trabajadores entre empresas y el crecimiento de los defectos de fabricación evidenciaron una crisis de productividad de este esquema, en el marco de un fuerte desgaste de la fuerza de trabajo derivado de su uso intensivo y de una nueva eficacia de la resistencia obrera que devino de la homogeneización y unificación de la clase. Para contrarrestar esta tendencia que comenzaba a poner en serio riesgo la acumulación de capital, se introdujeron modificaciones en la línea de montaje que permitieron relanzarla, sin abandonar los dos principios básicos que venían posibilitando su funcionamiento desde comienzos de siglo: la producción de flujo continuo y el fraccionamiento del trabajo (Coriat 2001).

Así, la línea de montaje abandonó la correlación entre un hombre, una tarea y un puesto, y se constituyeron equipos de trabajo pequeños donde las indicaciones sobre qué producir siguieron siendo asignadas por la dirección. La incorporación de estos equipos permitió la descentralización de algunas tareas y configuró un nuevo espacio de trabajo. También mutaron los atributos requeridos a la fuerza de trabajo y desde las gerencias comenzó a manifestarse la necesidad de operarios polivalentes, calificados y autónomos que dinamizaran el trabajo al interior de los equipos y distribuyeran las tareas libremente al interior de estos grupos. 
Pese a su relevancia, estos cambios no son más que una manifestación reciente de un largo recorrido de tensiones en la relación capital-trabajo que se remonta a los orígenes del modo de producción vigente.

Así, la primera gran transformación está dada por el paso de la producción artesanal a la producción capitalista. En el estadio precapitalista coexistían formas productivas artesanales en las que el maestro confeccionaba la totalidad de la mercancía y transmitía esos conocimientos a sus aprendices, con formas protocapitalistas, donde un "capitalista" entregaba insumos y medios de producción a trabajadores desconcentrados o a destajo que recibían un pago por pieza realizada (Smith 2001; Marx 1961). La producción capitalista propiamente dicha apareció con la concentración de un gran número de obreros (artesanos desposeídos) en un mismo espacio de trabajo que era propiedad de un capitalista individual quien, aunque poseía los medios de producción, carecía del conocimiento necesario para detentar el control del proceso productivo. La totalidad de la confección de la pieza continuó estando asignada a un mismo obrero-artesano, pero la aglomeración permitió incrementar la productividad al establecer el tiempo de trabajo social y avanzar en la división del trabajo considerando el conocimiento sobre el proceso productivo.

El taller así constituido, con crecientes niveles de división del trabajo, abrió paso a la producción de manufacturas. La extensión de la división de las tareas y su simplificación permitieron la incorporación de fuerza de trabajo menos calificada, especialmente proveniente de los desocupados que formaban el ejército de reserva. Esto permitió la constitución de una maquinaria humana ${ }^{7}$, fue trasladando el saber productivo hacia el capital e hizo posible el control de los tiempos de trabajo. De esta manera, se inicia una etapa en la que los trabajadores comenzaron a dejar de ser portadores del conocimiento productivo y se transformaron en portadores de un mero atributo que les permitía producir.

Con la división del trabajo fuertemente desarrollada, el siguiente paso fue la sustitución de trabajo vivo por capital. La maquinaria invadió el espacio de trabajo constituyéndose en la organizadora del proceso global, primero mediante la mecanización y luego a través de la robotización del proceso productivo. Así, el trabajador quedó subordinado a los tiempos de las máquinas y a las cadencias establecidas por las direcciones de fábrica. La mayor estandarización de los insumos derivados de estas formas productivas favoreció la integración vertical y la formación de grandes unidades productivas (Aglietta 1979; Coriat 2001). El elevado control de los tiempos de trabajo impactó nuevamente de manera muy fuerte en los niveles de productividad, vía la producción masiva de mercancías. Estas grandes empresas que fabricaban grandes volúmenes, controlaron gran parte de la producción pero, al mismo tiempo, engendraron las condiciones que definirían sus propias limitaciones. La resistencia sindical cobró vigor gracias a la aglomeración y a la homogeneización de clase y posibilitó la reducción de la brecha entre productividad y salarios, generando la necesidad de un nuevo esquema de explotación que recompusiera la rentabilidad empresaria.

7 "La maquinaria específica del período manufacturero sigue siendo el obrero colectivo mismo, formado por la combinación de muchos obreros parciales" (Marx 1961, 424). 
Las limitaciones técnico-económicas del sistema de trabajo parcelario y repetitivo condujeron a que la configuración productiva comenzara a mutar, tanto hacia adentro como hacia fuera de las unidades productivas. Al interior de la firma se observaron esquemas de trabajo en equipo, reducción de inventarios y nuevos atributos de la fuerza de trabajo. Hacia fuera, estas firmas comenzaron a deslocalizar y descentralizar algunas tareas en unidades más pequeñas y flexibles, transfiriendo riesgos y costos y conduciendo hacia una lógica de empleo dominada por el mercado (Boyer 1992; De la Garza 2000; Vidal 2013).

Las transformaciones en la línea de montaje y los procesos de descentralización productiva representan dos caras de un mismo proceso: la reconversión del aparato productivo. Este proceso fue acompañado por una polarización de las calificaciones y condiciones de trabajo según rama de actividad, tamaño de la empresa y puestos de trabajo, entre otros, y por una fuerte fragmentación del movimiento obrero que se reforzaba con la deslocalización y la tercerización. Ambas cuestiones derivaron en un fuerte deterioro de las relaciones laborales.

El fenómeno de encadenamiento en red de "empresas proveedoras de empresas" impacta en la configuración industrial, insertando la lógica de producción de servicios en la estructura de la producción industrial. En este marco, el proceso de "tercerización" (subcontratación) contribuye al proceso de "terciarización" (asociado al aumento de la importancia relativa de las actividades terciarias) y a su vez lo diversifica, acentuando la dificultad para precisar las características que adquieren los servicios desde el punto de vista de la organización del trabajo y de las características que pueden o no asimilarlo al trabajo industrial (Vidal 2013; Gadrey 1990). El entramado de redes de empresas implica también un cambio sustantivo en los ambientes en los que se despliega cotidianamente el trabajo, en particular en las condiciones laborales que exceden lo contractual e impactan en las actividades, los oficios, las posibilidades de desarrollo de los trabajadores y, muy especialmente, en la construcción de la identidad profesional.

A partir de los setenta se da un desarrollo productivo que, para algunos autores, implica la conformación de grupos de trabajos multifuncionales, con elevada calificación del obrero y dinámicas de control de calidad gestionadas por el grupo, bajo una lógica de "primero vender, luego producir" (Coriat 1997). En otros casos se enfatiza el surgimiento de estrategias de la empresa apostando a la flexibilidad y al involucramiento (Lipietz 1994). En cualquier caso, casi todos los autores señalan la centralidad del trabajador polivalente en el marco de un grupo de trabajo, donde la organización fija su objetivo en función de la productividad del sistema, en vez de tomar en cuenta la productividad individual. Siguiendo a Coriat (1997), el nuevo escenario de trabajo automatizado acentúa la importancia de la polivalencia y la calificación del obrero, requiere más responsabilidad y compromiso del obrero y disminuye el trabajo directo al mismo tiempo que incrementa las tareas indirectas (mantenimiento de equipos, calidad, gestión, etc.).

Este proceso implica una cierta recuperación del saber productivo por parte de la clase trabajadora, aunque no es el conocimiento productivo integral típico de la artesanía. Por el contrario, se trata de un conocimiento que ha sufrido las mutaciones enfrentadas por el proceso productivo y que vuelve al trabajador de manera fragmentada. Nuevamente, esta dinámica responde a la propia 
necesidad del capital de intentar recomponer la productividad en el marco del nuevo proceso de trabajo. Por ello Coriat (1997), como si le diera lecciones al capital, describe un esquema donde la devolución de ese saber productivo debe acompañarse de un mecanismo que genere mayores niveles de implicación en los trabajadores con los objetivos de las empresas.

La automatización requiere, en términos generales, mayores niveles de calificación en la fuerza de trabajo. Sin embargo, ante la heterogeneidad de estos procesos, el grupo de trabajadores que se ve fortalecido por una creciente calificación y mejores condiciones de trabajo coexiste con una masa significativa de trabajadores que pierden atributos productivos, dándole continuidad al proceso de descalificación iniciado anteriormente.

\subsection{Cambios operados al interior del proceso de trabajo}

Si bien todas las formas de organización de la producción descritas emergen como un paradigma organizativo a fines de los setenta y principios de los ochenta, algunas de estas ya estaban presentes desde los primeros años de la segunda posguerra. Incluso, varias de estas se presentaban fuertemente interrelacionadas entre si, lo que dificulta una caracterización diferenciada.

A estas nuevas prácticas productivas, la literatura socioeconómica las denominó postfordistas, en contraposición a las vigentes desde la instauración de la línea de montaje, asociada a lo que la escuela de la regulación llamó fordismo y dio lugar al régimen de acumulación de la producción en masa vía la estandarización. A su vez, la línea de montaje coexistió con formas pretéritas de organización del trabajo basadas en el control sobre el proceso productivo mediante la escisión entre la concepción y la ejecución (taylorismo), con el objetivo de incrementar la intensidad del trabajo y, consecuentemente, la productividad.

Una de las principales características de estas nuevas formas organizacionales es la producción justo-a-tiempo (just-in-time, JIT), también llamada toyotismo/ohnismo por haber sido aplicada por primera vez en la empresa japonesa según las ideas de Ohno Taiichi. El objetivo de esta práctica productiva es la eliminación de las existencias reguladoras (inventarios). Para lograrlo se trata de ajustar la producción a las necesidades de venta, lo cual permite minimizar los requerimientos de capital y espacio destinados a almacenamiento no solo de los productos finales, sino también de los insumos y de las piezas intermedias. Dado que la reducción de existencias eleva el riesgo implícito en el sistema productivo, esta práctica aparece fuertemente asociada a la eliminación de errores durante el proceso productivo y al control de la calidad (Sayer 1986; Coriat 1992b; Neffa, Panigo y López 2010). Pero al mismo tiempo, estos esquemas productivos generan tensiones en la producción asociadas a la falta de existencias que causa inconvenientes y presiones sobre los trabajadores, ante las eventualidades que surgen en el marco del contexto productivo (Slaughter 1998).

El sistema JIT tiene fuertes impactos dentro y fuera de la empresa, ya que esta configuración productiva implica una red de empresas subsidiarias (Finkel 1994), proveedores y subcontratistas que, o bien operan bajo su misma lógica, o están en condiciones de adecuarse a la dinámica propuesta por la empresa principal. Esta articulación puede ser conflictiva ya que las grandes decisiones 
(tiempos, calidad, etc.) se concentran en la corporación. Coriat (1992a) ${ }^{8}$ sostiene que las relaciones de subcontratación que han generado las nuevas prácticas productivas se caracterizan por i) la estabilidad de largo plazo, ii) los vínculos institucionalizados y jerarquizados, iii) la contractualización, y iv) el favorecer a la internalización de la innovación.

Por su parte, la eliminación de desperdicios significa la búsqueda de la calidad total en el proceso productivo, para lo cual se requieren crecientes niveles de compromiso de los actores intervinientes. Esta dinámica generalmente se complementa con el trabajo en equipo, cooperativo, en círculos de calidad, donde se discuten los problemas y se proponen soluciones. En este marco de trabajo conjunto se pregona una suerte de dilución de jerarquías entre trabajadores manuales e intelectuales.

Los crecientes niveles de compromiso necesarios en el marco de este modelo de producción se logran a partir de proporcionar a los trabajadores espacios de participación más amplios en la organización del proceso productivo. Esta participación puede asumir, según Finkel (1994), dos formas fundamentales: la democracia industrial o los círculos de calidad. Ambos esquemas se presentan como alternativas a la organización científica del trabajo pero con objetivos distintos. Por un lado, el primero de ellos se orienta a generar una "higiene mental positiva" en la vida industrial. Esta perspectiva considera la existencia de un sistema socio-técnico compuesto por un entorno técnico y por un entorno social, y sostiene que para que el sistema funcione bien técnicamente y se eviten problemas (ausentismo, accidentes laborales, baja productividad), deben atenderse las cuestiones del entorno social, corriendo la mirada desde el individuo hacia el grupo. Por su parte, los círculos de calidad (o kaizen), al menos inicialmente en Japón, no intentaron motivar a los empleados, sino identificar, resolver y descentralizar problemas de control de calidad mediante el trabajo en equipo de obreros, técnicos e ingenieros.

Los sistemas productivos que operan a través de equipos de trabajo generan las condiciones para que exista una mayor implicación de los trabajadores con la empresa y sus objetivos de producción. Al mismo tiempo, a diferencia del modelo de integración vertical, el perfil del trabajador ya no requiere especialización sino cierto nivel de calificación que permita asumir distintas tareas (polivalencia) que son asignadas al grupo y se distribuyen dentro del mismo. Pese a su relevancia, estas estructuras participativas pueden coexistir con formas no participativas de organización de la producción en la rama, en la industria, e incluso, en el mismo establecimiento.

La descripción anterior pone en evidencia que las distintas formas de organización de la producción han surgido, en parte, como respuesta a las necesidades de los capitalistas de incrementar los niveles de productividad. Así, las lógicas de la organización del trabajo que dominan en distintos periodos del desarrollo capitalista pueden ser vistas como un avance del capital sobre el trabajo, que surge a partir de la búsqueda de incrementos en la tasa de ganancia y como un mecanismo que garantiza el restablecimiento del control ejercido por los capitalistas

8 El autor describe cómo, en los ochenta, la tercerización en Toyota alcanza al 70\% del valor total de los vehículos por hora. 
sobre el proceso productivo. Sin embargo, este proceso está condicionado por el accionar obrero que limita el nivel de explotación capitalista. Esta dinámica, que tiene como eje la administración del conocimiento del trabajador sobre el proceso productivo, funda una relación conflictiva entre capital y trabajo.

A partir de la descripción de la evolución y las formas que adquiere el proceso de trabajo, de las diferentes formulaciones realizadas sobre las condiciones que enmarcan la actividad productiva desarrollada por los trabajadores, cobra sentido preguntarse cuáles son las relaciones que pueden establecerse entre las particularidades de las formas vigentes de organización productiva, y la dimensión referida a la forma de gestión del trabajo como aspecto relevante para la configuración de la calidad del trabajo.

\section{El análisis de las condiciones laborales desde la perspectiva de la calidad del empleo}

\subsection{Aportes y limitaciones de diferentes enfoques sobre las condiciones laborales}

El análisis de las condiciones laborales ha sido abordado desde distintas perspectivas que utilizan una amplia variedad de metodologías y circunscriben el campo de análisis de distintas maneras, aunque muchas veces compartiendo dimensiones. Así, podemos identificar un extenso trayecto asociado a una evolución conceptual que culmina en la definición de la calidad del empleo como un término que recoge dimensiones de análisis y herramientas metodológicas propias de distintos abordajes.

En el caso particular de Argentina, observamos que los avances sobre el tema se articulan, al menos parcialmente, con los cambios que se fueron sucediendo a nivel internacional, los cuales fueron incorporando o relegando dimensiones a medida que se profundizaba en el estudio sobre esta temática.

En la década del ochenta tuvo lugar el enfoque renovador que, en Argentina, se plasmó en la adopción de normativas vinculadas a las condiciones y el medioambiente de trabajo, las cuales se conocieron bajo la sigla CyMAT. A diferencia de la antigua concepción de higiene y seguridad que ponía el acento fundamentalmente en las tareas manuales y de ejecución, las CyMAT incluyeron múltiples dimensiones que no solo se relacionan con el cuidado de la salud de los trabajadores y la resolución de conflictos derivados de esta problemática, sino también con las actividades de prevención tendientes a reducir o limitar los riesgos laborales. Asimismo, las CyMAT reconocieron la importancia creciente de los procesos de creación y transmisión de conocimiento y de las tareas de producción de mayor complejidad en términos de conocimientos y capacidades requeridas, así como también la relevancia de las actividades indirectas de servicios, administración y gestión en los diversos sectores de la economía. El eje central de las CyMAT se sustentó en la influencia de estas normas sobre la eficiencia de las empresas y las organizaciones, dado que su implementación conduce a mejoras de competitividad. En este sentido, las buenas condiciones de trabajo impactarían positivamente en el incremento de la productividad, en la mejora 
de la calidad de los bienes y servicios producidos, en la reducción de los costos y en el mayor compromiso de los trabajadores. Las dimensiones contempladas en las CyMAT están estrechamente vinculadas a las características que adoptan los procesos de trabajo y se desprenden del modelo analítico formulado por el Centro de Estudios e Investigaciones Laborales (CEIL) (Neffa 1995).

Hacia finales de la década del ochenta y principios del noventa, la temática de las condiciones de trabajo comenzó a ser abordada bajo la óptica de la informalidad laboral y del trabajo precario (TP). La perspectiva de la informalidad laboral se focaliza en dos situaciones diferentes. Por un lado, se asocia con los trabajadores que se desempeñan en los sectores económicos atrasados, marginales o de baja productividad (Lindenboim 1990). Por otro lado, segmenta a los ocupados en función del registro de la relación de trabajo, por lo que la informalidad aparece como propia de aquella actividad laboral que se realiza por fuera del marco normativo legal (no registrado) (Novick, Mazorra y Schleser 2008).

Por su parte, los análisis sobre precariedad se basan en la caracterización de esta situación en contraposición a la del empleo típico que toma la forma de la relación salarial de tiempo completo, con un único e identificable empleador, con una contratación por tiempo indeterminado, realizado en el domicilio del empleador y protegido por la legislación laboral y la seguridad social (Feldman y Galin 1990). Por ello, se considera empleo precario al que es realizado en un plazo determinado, de manera eventual, subcontratado o a domicilio, clandestinamente o no registrado, así como también al empleo asalariado oculto bajo formas de relación no salariales (por ejemplo, el cuentapropismo). Los estudios sobre precarización se concentraron en el trabajador y no en el puesto de trabajo, por lo que el interés por analizar el TP a partir del relevamiento de los asalariados y desocupados a nivel agregado condujo a dos modificaciones claves en torno a las dimensiones consideradas en las CyMAT. Por un lado, se dejó de considerar la importancia de la organización del trabajo para el desarrollo de capacidades en los trabajadores y, por el otro, se introdujo la tercerización como mecanismo relevante para el estudio del fenómeno de la precariedad laboral.

Durante los primeros años del nuevo siglo se instaló en el país una nueva categoría analítica, la de trabajo decente (TD), cuyos antecedentes se remontan, en el plano internacional, hacia la segunda mitad de la década del noventa. Bajo esta nueva categoría se buscó desarrollar un marco para el diseño de programas orientados a mejorar las condiciones de empleo e ingresos, la protección social y el fortalecimiento del diálogo social. Así, el TD se incorporó como tema de agenda con el objetivo de reducir su déficit, enfatizando en las condiciones necesarias para la promoción del trabajo productivo, es decir, aquel considerado como justamente remunerado, en condiciones de libertad, equidad, seguridad, dignidad y en el cual los derechos eran respetados. En el ámbito local se incorporó la temática del TD en el plano de las políticas públicas mediante la creación del Programa Nacional de Trabajo Decente que permitió incluir el concepto en la normativa laboral, instalarlo en el discurso oficial. En este marco, a partir de 2004 se fueron desarrollando en el país algunos estudios tendientes a proveer información sobre la situación laboral local, especialmente como iniciativa del Grupo de Estudios del Trabajo (GrET) en el marco del Programa Nacional de TD recién mencionado. Actualmente, si bien se reconoce la potencia del concepto 
de Trabajo Decente cuando se busca situar la problemática en la agenda de diversos actores institucionales y decisores gubernamentales, también se asume que dicha categoría resulta limitada desde el punto de vista del estudio integral del fenómeno (Mocelin 2008).

La descripción de las distintas perspectivas pone de manifiesto las limitaciones existentes en estos conceptos en relación con las dimensiones de análisis retomadas. En general, se consideran especialmente las cuestiones jurídicoinstitucionales que definen las características de la relación laboral (modalidad de contratación, registro, nivel de ingresos, extensión de la jornada, protección social, etc.), las cuales impactan en el trabajador fundamentalmente en términos económicos. Si bien estas dimensiones son suficientes para dar cuenta de algunas características de las condiciones de trabajo, presentan limitaciones para describir otros aspectos que surgen de la dinámica laboral, entre ellos el proceso de desarrollo profesional y personal de los trabajadores. A su vez, estas perspectivas no contribuyen a describir y explicar los cambios evidenciados a lo largo de tiempo en el proceso de trabajo ya que no analizan los distintos rasgos que adopta la organización productiva y/o el impacto de esta sobre la calidad de vida del trabajador.

En este contexto, en la actualidad, el interés se centra en el concepto de Calidad del Empleo (CE), tanto en el ámbito de las políticas públicas como en el académico. La noción de CE comenzó a ser ampliamente utilizada desde principios del 2000 por ser considerada una categoría que, a diferencia de las ideas sobre trabajo precario y trabajo decente, contempla la perspectiva de los empleadores - quienes focalizan su atención en los aumentos de productividad-, la de los trabajadores - quienes se expresan a partir de los sindicatos- y la del Estado - interesado en formular políticas públicas que favorezcan la calidad de empleo, tanto desde el punto de vista de las políticas de empleo como de las de salud-. En términos generales, los estudios sobre calidad del empleo se estructuran en base a tres ejes analíticos principales que consideran: i) la perspectiva de los agentes, ya sea el Estado, las empresas o los trabajadores; ii) el nivel de agregación del análisis, centrado en el puesto de trabajo o en el empleo como variable agregada, y iii) las dimensiones utilizadas, en particular, las subjetivas y objetivas (Farné 2003). Entre estas últimas se destacan el nivel de ingresos y las modalidades de contratación, aunque también se fueron incorporando paulatinamente otras tales como las condiciones de trabajo, la salud, los derechos del trabajador, los aspectos vinculados con el diálogo social, los logros personales y la autorrealización.

\subsection{Las condiciones laborales desde la perspectiva de la calidad del empleo}

Los análisis relacionados con la calidad del empleo han adquirido centralidad en las últimas décadas aunque queda claro que, utilizando diferentes denominaciones, se ha discutido en torno a esta problemática desde los orígenes de la industrialización. El énfasis actual puesto en esta temática surge al reconocer que el desempleo no es el único ni necesariamente el más importante de los problemas que afectan al empleo. 
La reconocida importancia de estudiar las condiciones laborales desde la perspectiva de la calidad no anula el hecho de que el concepto de calidad de trabajo y/o de empleo se encuentra aún en construcción (Champlin 1995; Ermida 2001; Farné 2003; Lanari 2005) y que su uso reviste importantes grados de complejidad derivados del interés de distintos agentes relacionados con la temática, de la diversidad de contextos de aplicación y de las distintas dimensiones incluidas en la definición.

A pesar de las diferencias establecidas por distintos autores, en general las definiciones aportadas coinciden en destacar la importancia del bienestar individual y social del trabajador. En este marco, por ejemplo, Fernández (2003) define a la calidad del empleo como "todos aquellos aspectos relacionados con el empleo que tengan o puedan tener algún efecto sobre la calidad de vida del trabajador, tanto dentro como fuera del trabajo" (2003, 1), mientras que Reinecke y Valenzuela (2000) sostienen que la calidad del empleo "se puede definir como el conjunto de factores vinculados al trabajo que influyen en el bienestar económico, social, psíquico y de salud de los trabajadores" $(2000,30)$.

Los distintos actores involucrados en el proceso de trabajo enfatizan en diferentes aspectos que definen a la calidad del empleo. Cuando se considera al Estado y la política pública, la calidad del empleo se evalúa a partir del nivel educativo y de la experiencia laboral. Para las empresas, la calidad se define en función de sus objetivos de productividad y versatilidad $y$, en este sentido, resultan relevantes las cuestiones vinculadas con las calificaciones de los trabajadores y sus capacidades de autonomía y flexibilidad. Desde la perspectiva de los trabajadores y sus organizaciones, se consideran además de las cuestiones mencionadas anteriormente, aquellas asociadas con las prestaciones sociales y la estabilidad laboral (Farné 2003; Lanari 2006).

También existen limitaciones para la construcción del concepto derivadas de la diversidad de contextos de aplicación. Esto incluye las diferencias relacionadas con la actividad manufacturera versus la de servicios, pero también una amplia variedad de situaciones vinculadas con el tipo de relación laboral implicada. A modo de ejemplo, las consideraciones realizadas por la Organización Internacional del Trabajo (OIT) con relación al trabajo "decente" incluyen no solamente al empleado asalariado, sino también a cualquier persona que desarrolla actividades por cuenta propia o que trabaja bajo las nuevas modalidades de contratación introducidas a partir de los regímenes de flexibilización laboral. La diversidad de situaciones de trabajo que pueden ser definidas a partir de estas especificidades pone de manifiesto la imposibilidad de contar con una única forma de aproximación a la calidad del empleo (Barreto 2001; Farné 2003; Findlay, Kalleberg y Warhust 2013).

A lo anterior se adiciona, tal como destaca Vidal (2013), la diferencia entre la realidad que enfrentan diferentes grupos de trabajadores en un mismo lugar de trabajo. Por una parte, se encuentran aquellos que poseen elevados niveles de calificación y autonomía; por otra, quienes cuentan con bajos niveles de calificación $y$, debido a esto, enfrentan trabajos de muy alta intensidad o fuertemente acotados. Así, quienes se encuentran en estas últimas situaciones afrontan y evalúan las condiciones de calidad de empleo de manera muy diferente que los primeros.

En lo que respecta a las dimensiones consideradas para evaluar la calidad del empleo, observamos que cuestiones tales como las condiciones y medioambiente 
de trabajo, los derechos del trabajador, los aspectos vinculados con el diálogo social, los logros personales y la autorrealización son elementos retomados en la mayor parte de los análisis relevados (Rosenthal 1989; Gittleman y Howell 1995; Verdera 1995; Infante y Vega-Centeno 1999; Beatson 2000; citados en Farné 2003). Otros autores (Reinecke y Valenzuela 2000; Van Bastelaer y Hussmann 2000; Rodgers y Reinecke 1998; Rodgers 1997; Findlay, Kalleberg y Warhust 2013), por su parte, agregan a los anteriores aspectos tales como las modalidades de contratación y la flexibilidad en la programación de actividades y en la gestión de las horas de trabajo (Findlay, Kalleberg y Warhust 2013; Loughlin y Murray 2013).

A nivel nacional, pueden identificarse recientemente un conjunto de trabajos que tienen como objetivo fundamental realizar un aporte sobre las dimensiones relevantes para el análisis de la calidad del empleo y, al mismo tiempo, a partir de estas dimensiones, describir la realidad de Argentina en relación con esta temática (Rodríguez et al. 2012; Amorín y Erbes 2007), tanto desde la perspectiva de las empresas (Erbes y Amorín 2009; Amorín et al. 2007) como desde la de los trabajadores (Pujol et al. 2012; Roitter et al. 2013).

En este marco, Roitter et al. (2013) señalan que el análisis de la calidad del empleo requiere considerar distintos factores que pueden ser agrupados en tres grandes dimensiones: la institucional, la de la organización productiva y la gestión del trabajo y la de la representación sindical. Estas dimensiones y sus factores constitutivos tomados conjuntamente permiten dar cuenta de una tipología que sintetiza distintos niveles de calidad del empleo.

La dimensión institucional incluye a todos aquellos factores que caracterizan la relación laboral y que suelen ser definidos por la legislación, por ejemplo, a partir del cumplimiento de la Ley de Contrato de Trabajo. Se trata de una dimensión que incluye información sobre el contrato laboral, la remuneración, la duración, la intensidad y el ritmo de la jornada y el medioambiente de desarrollo laboral. La dimensión vinculada con la organización productiva y gestión del trabajo pretende captar los rasgos que definen el nivel calificante de la estructura de producción y se analiza a través de factores tales como los procesos de adquisición de capacidades, las formas de realizar la tarea, la autonomía del empleado para el desarrollo de su actividad, la forma de participación de los trabajadores, la flexibilidad horaria y funcional y el sistema de control y/o evaluación de desempeño que se implementa. La tercera dimensión es la de la representación sindical y da cuenta de la relación que tiene el trabajador con el sindicato, a partir de la existencia de delegados sindicales en la empresa y de la forma de participación de los trabajadores en la acción colectiva.

La revisión de la literatura nos permite sostener que el tratamiento de la calidad del empleo se ha concentrado preponderantemente en aquellos factores que se asocian con las dimensiones institucional y de representación sindical. Por el contrario, aquellos aspectos vinculados con la organización productiva y la gestión del trabajo han sido enfatizados con menor frecuencia y profundidad. Esto se relaciona con la complejidad en la identificación y medición de los factores incluidos en esa dimensión, tanto si se consideran a nivel de empresa como del trabajador. Es sobre esta cuestión que este trabajo se propone realizar un aporte en las próximas secciones. 


\subsubsection{La organización del trabajo como dimensión de análisis de la calidad del empleo}

Erbes, Roitter y Kababe (2014) realizan una detallada descripción de los distintos factores incluidos en la dimensión de la organización productiva y la gestión del trabajo para comprender la dinámica de los procesos de aprendizaje. Esta caracterización es útil también para la definición de diferentes niveles de calidad del empleo, particularmente en el marco de las formas más recientes de organización de la producción adoptadas por las empresas.

El primero de los factores considerados se asocia con los rasgos de los equipos de trabajo y considera las cuestiones más elementales de la definición del proceso productivo al analizar si este se desarrolla individual o colectivamente. Se trata de uno de los aspectos centrales de la dinámica formativa de las empresas, por cuanto el trabajo grupal, como herramienta social, permite que la actividad adquiera características enriquecedoras en la medida que promueve intercambios entre los trabajadores a partir de las experiencias compartidas. Sin embargo, la mera existencia de equipos de trabajo no es suficiente para favorecer los procesos de aprendizaje, sino que también es necesario considerar las características de estos equipos en términos de las tareas y responsabilidades que se les asignan al interior de los mismos.

Por su parte, la estrategia de la firma en lo referido al desarrollo de competencias se asocia con la manera en la que se efectiviza la adquisición de conocimientos, competencias y experiencias por parte del personal, tanto a través de las estrategias explícitas de formación -externamente y en el puesto de trabajo- y de los sistemas de capacitación, como de los planes de rotación de personal. Los procesos de capacitación y de formación externa pueden caracterizarse por distintos niveles de formalidad, inclusión de los trabajadores y especificidad de las temáticas abordadas (Grimshaw et al. 2002), dando lugar a un gradiente de situaciones que abarca desde los programas de formación general y continua, hasta aquellos específicos y puntuales encarados con el objetivo de desarrollar capacidades específicas. Por su parte, la adquisición de experiencias mediante la formación en el puesto y la dinámica de rotación se logra a través de la realización de distintas tareas, las cuales requieren planificación e implican niveles crecientes de complejidad de las actividades y de las calificaciones. De manera similar a lo expuesto en relación con la capacitación, estos procesos también dan lugar a la emergencia de un trabajador polivalente, pero a diferencia del caso anterior, aquí la polivalencia tiende a ser enriquecedora si promueve la aparición de nuevos conocimientos colectivos que impliquen una ampliación de los conocimientos individuales. Las estrategias de formación y los sistemas de capacitación son procesos sistémicos, que se retroalimentan mutuamente y, en este sentido, raramente pueden dar cuenta de manera aislada de la dinámica de adquisición de capacidades dentro de una firma. Lo anterior, no quita que ante necesidades específicas de construcción de habilidades individuales y/o rutinas organizacionales un mecanismo adquiera preeminencia por sobre el otro.

La autonomía se asocia con un conjunto de aspectos que se traducen en una competencia transversal necesaria para la profesionalización de cualquier ocupación (Zarifian 1996) y remite tanto a la actitud y a la posición que asume el 
trabajador frente al trabajo prescripto, como a la actitud esperada por la empresa en relación con estas cuestiones. Se trata de un factor que permite diferenciar un gradiente de situaciones en cuyos extremos se ubican el desarrollo estricto de los procesos de trabajo de acuerdo a las normas preestablecidas, o la posibilidad del trabajador de prever problemas e intervenir correctivamente sobre los mismos, aún sin contar con una supervisión directa. Así, no cualquier contexto favorece el desarrollo de mayores niveles de autonomía sino que, por el contrario, estos solo son posibles cuando: a) el trabajador dispone de cierta expertise para participar en las decisiones que atañen a las rutinas de su sector; b) los estilos de conducción imperantes son más formativos que directivos, y c) existe un bajo nivel de disciplina de las interacciones y el ambiente físico y social del trabajo que estimula la interconsulta y la cooperación.

El cuarto factor evalúa la participación y el involucramiento de los trabajadores desde dos perspectivas complementarias. Incluye tanto la actitud de la empresa hacia la participación de los empleados - por ejemplo, la receptividad frente a las propuestas-, como la propensión de los empleados a realizar aportes que permitan mejorar sus procesos de trabajo y cumplir los objetivos definidos por la firma. La inclusión de los trabajadores en equipos de mejora o en círculos de calidad ha sido vista, en las décadas más recientes, como la forma consolidada de participación de los trabajadores. Sin embargo, en el análisis de esta dimensión es importante no desconocer la relevancia de otras formas de participación que implican un nivel de acceso diferencial a la información y que conviven con formas menos participativas de gestión (Finkel 1994).

Un quinto factor que permite describir la forma en la que se organiza el trabajo es la flexibilidad. Esta se asocia a diversas cuestiones que afectan de distinta manera la calidad de empleo del trabajador. Por una parte, se refiere a la existencia de contratos de trabajo "no standards", de medio tiempo y por tiempo determinado, frente a aquellos que implican trabajo de tiempo completo y por tiempo indeterminado. Por otra parte, la flexibilidad se asocia a la tendencia relativamente reciente hacia el aumento de la capacidad con la que cuentan los trabajadores para desarrollar eficientemente distintas tareas en momentos específicos que son definidos por las necesidades de la organización y del mercado.

En lo que respecta al primer tipo de flexibilidad, existe acuerdo en torno a que la inseguridad en el empleo es una fuente de stress que involucra temor y ansiedad (Greenhalgh y Rosenblatt 1984, citado en Loughlin y Murray 2013) y que se asocia claramente con empleos de baja calidad. Sin embargo, la existencia de contratos de tiempo parcial no posee una relación tan clara con la calidad del empleo, en tanto es necesario considerar la congruencia de esta situación con los deseos del empleado. De esta manera, la incongruencia entre los deseos del trabajador y su situación laboral, ya sea como trabajador de tiempo completo o part-time, se vincula más claramente a la calidad del empleo que la existencia de distintos tipos de empleos definidos a partir de su duración (Loughlin y Murray 2013; Barnett 2006; Hock y DeMeis 1990, ambos citados en Loughlin y Murray 2013).

En relación con el segundo tipo de flexibilidad, Bosch y Lehndorff (2001) lo introducen como una dimensión clave que se define en función de la orientación de las actividades hacia el mercado, de las características particulares de la demanda, de la mayor o menor asignación de responsabilidades entre 
los trabajadores y del nivel de calificaciones que es requerido. Es posible distinguir un tipo de flexibilidad pasiva u horaria, de otra activa o funcional. El primer tipo resulta habitual en organizaciones que requieren la disponibilidad de los trabajadores en horarios y bajo esquemas de trabajo no siempre convencionales. Se trata de una flexibilidad de tipo numérico y que responde a puestos individuales con tareas estandarizadas, donde los trabajadores pueden ser reemplazados en cualquier momento y la dotación de personal puede variar considerablemente en concordancia, fundamentalmente, con la demanda del mercado. Por su parte, la flexibilidad activa se manifiesta cuando las firmas no están dispuestas a prescindir del conocimiento, las habilidades y la experiencia individual de sus trabajadores y, en consecuencia, se apoyan en las capacidades de sus empleados para auto organizarse y gestionar su tiempo de trabajo. Este tipo de flexibilidad funcional permite dar respuesta a los requerimientos del mercado en términos de una fuerza de trabajo con elevada calificación y gran disponibilidad horaria.

El sexto factor retomado considera distintas maneras de control y/o evaluación de las actividades y el desempeño de los trabajadores. En general, las características que asume este factor se materializan en los dispositivos organizacionales desarrollados para garantizar el cumplimiento de los objetivos previstos por la empresa. En particular, es posible considerar la existencia de distintos esquemas que abarcan desde el seguimiento jerárquico directo de la actividad de los trabajadores, hasta mecanismos indirectos-externos — vía evaluación por parte de pares- o de autocontrol, en los cuales lo que se busca garantizar es el logro de los objetivos a partir del involucramiento de los trabajadores pero sin incidir directamente en la forma en la que estos desarrollan sus actividades. En este sentido, Edwards (1979) destaca la emergencia de formas estructurales de control, asociadas a la estructura organizativa del trabajo por oposición a las formas personalizadas y, en la misma dirección, Burawoy (1989) señala la importancia de lo que él llama el 'individualismo competitivo' como elemento que permite la internalización del control.

Complementariamente a los factores identificados por Erbes, Roitter y Kababe (2014), es necesario considerar también las modalidades de gestión que la empresa aplica para promover el cuidado de la salud y la seguridad de los trabajadores, lo que no remite solo al cumplimiento de las leyes y las normas existentes, sino que involucra una gestión que tienda a evitar accidentes y enfermedades profesionales y a promover bienestar y seguridad en los ámbitos de trabajo, con la participación activa de los trabajadores y sus representantes.

En función de lo anterior, sostenemos la relevancia de la organización productiva y la gestión del trabajo como un elemento fundamental para analizar la calidad del empleo, especialmente a la luz de los cambios en los modelos productivos evidenciados en las últimas décadas. Estos nuevos modelos productivos, instalados fundamentalmente con el objetivo de aumentar la eficiencia productiva y la competitividad de las firmas, implican en la actualidad nuevas demandas sobre las habilidades, los conocimientos y las capacidades de los trabajadores, con impactos sobre su calidad del empleo y de vida que son, al menos, discutibles. Estas discusiones son objeto de la próxima sección. 


\subsubsection{Organización del trabajo y calidad del empleo en el nuevo contexto productivo}

La dimensión de la organización del proceso productivo y la gestión del trabajo, junto con las dimensiones institucional y de representación sindical, permite dar cuenta de diferentes niveles de calidad del empleo. En particular, se trata de una dimensión que define un conjunto de elementos capaces de explicar el desarrollo de procesos de aprendizaje que revisten una doble importancia. Por un lado, potencian la dinámica productiva y competitiva de la empresa en tanto facilitan la innovación. Por el otro, generan oportunidades laborales que permiten el crecimiento profesional y personal de los trabajadores. Si bien ambos objetivos pueden concatenarse y promoverse simultáneamente, vastos segmentos de la literatura especializada han concluido que la implementación de ciertas dinámicas asociadas a los nuevos modelos de producción genera beneficios para la empresa que no se traducen necesariamente en una mejora en el bienestar del trabajador. Esto ha conducido a importantes debates sobre la relevancia y características que deben adquirir los distintos factores que dan cuenta de la organización del trabajo para dotar a los empleos de mayor calidad $\mathrm{y}$, por lo tanto, de una mejor calidad de vida al trabajador.

Así, cada uno de los factores anteriormente descritos se manifiesta de manera diferencial en el marco de los distintos modelos productivos que se han sucedido históricamente, con un impacto también heterogéneo en la determinación de niveles variables de calidad del empleo.

La emergencia de los nuevos modelos productivos pos década del setenta se ha sustentado ampliamente en el ascenso de la relevancia de los equipos de trabajo por sobre el trabajo individual —característico del modelo fordista-, lo cual requiere, al menos, una resignificación de las dinámicas productivas colectivas. El trabajo en equipo se presenta como un espacio de construcción grupal de saberes, donde la cercanía física con el otro y el sentido de pertenencia al grupo facilitan el desarrollo de procesos de aprendizaje. Sin embargo, la mera existencia del equipo no garantiza el surgimiento de estas dinámicas. Por el contrario, se requiere analizar si estos promueven o no el protagonismo de los trabajadores en actividades de concepción del proceso productivo y si se consideran aspectos más específicos de las interacciones, entre ellos los vínculos directos o indirectos que establece cada trabajador con las actividades de producción (Batt 2004). Por consiguiente, no siempre el trabajo en equipo garantiza mayores niveles de calidad del empleo sino que, por el contrario, puede generar lógicas de trabajo en las que la rutinización de las tareas operativas conduzca a la insatisfacción del trabajador como consecuencia de las escasas posibilidades de aprendizaje y desarrollo profesional en el marco del grupo de trabajo. A su vez, el trabajo en equipo puede tornarse un instrumento para facilitar el control de la productividad en el marco de mediciones grupales, dado que, ante incentivos económicos asociados al rendimiento grupal, se produce un mayor control desde los pares sobre el trabajo y la asistencia de sus compañeros.

En este sentido, algunos autores evidencian una importante preocupación por la existencia de procesos de descolectivización del trabajo asalariado (Linhart 2010; Bacon y Storey 1994). He aquí una paradoja clave: en las formas de 
organización que adquiere actualmente el trabajo, la fragilización de los colectivos de trabajo es por contraste tan importante como los discursos sobre la cooperación, el involucramiento de todos y el desarrollo del sentido de equipo. De este modo, se observa en las empresas e instituciones un des-apuntalamiento del lazo social y profesional que genera "un individualismo autista o una desubjetivación adaptativa" (Diet 2010, 240) que se encuentra en el origen del sufrimiento en el trabajo. Se trata de situaciones en las que se refuerza la lógica instrumental y la organización aprovecha la vulnerabilidad del trabajador para ganar aún más en productividad siempre apoyándose en los preceptos del "buen desempeño". Así, algunas características comunes de la organización del trabajo contemporáneo operan en desmedro de la constitución de colectivos de trabajo y a la vez activan procesos de desubjetivación en los trabajadores (Pujol y Hoyos 2013; Diet 2010).

También los procesos vinculados con la adquisición de competencias y calificaciones han evidenciado transformaciones en los últimos tiempos. En particular, uno de los cambios más sobresalientes se relaciona con la importancia asignada al desarrollo de capacidades consideradas transversales y más vinculadas a rasgos personales - capacidad de trabajar en equipo, de resolver problemas, de comunicarse-, en detrimento de aquellas que se logran a partir de la escolaridad o de la misma dinámica de formación en el puesto de trabajo. Como consecuencia de esto, las competencias técnicas de los operarios ya no ocupan el único -y tal vez tampoco el más importante- lugar en la generación de mayores niveles de productividad y competitividad en las firmas. En este contexto, se observan procesos en los cuales el objetivo es dotar a los empleados de habilidades que les permitan ocupar diferentes posiciones dentro de la organización de manera tal que se garantice el flujo continuo de trabajo. Esto redunda en programas de formación continua que abarcan a una amplia proporción de los trabajadores de las empresas y que están relacionados con temas generales y de escasa complejidad (Grimshaw et al. 2002), lo cual se contrapone con la existencia de programas sistemáticos para la formación y la capacitación, abarcan a grupos específicos y se centran en temáticas relevantes para estos actores.

Tal como sostuvimos en la sección anterior, el impacto más directo de estas prácticas relacionadas con la adquisición de experiencias es la emergencia del trabajador polivalente. Esto puede tener un impacto positivo o negativo sobre los niveles de calidad del empleo, asumiendo características virtuosas cuando la formación que se provee al trabajador — tanto a partir de la rotación como de la capacitación- supone la aparición de conocimientos individuales y colectivos nuevos (Méhaut 1994) y, en este sentido, genera una ampliación de los conocimientos de los operarios. En este marco, la evaluación de la potencialidad de los empleos para desarrollar capacidades en los trabajadores supone abordar, al menos, tres actividades centrales, a saber: a) determinar las capacidades o competencias requeridas por cada función productiva y los niveles de logro esperados; b) sistematizar dichas funciones en una matriz que permita "mapear" las competencias existentes en un área o sector y gestionar su desarrollo, y c) evaluar las competencias de manera periódica y decidir acciones que favorezcan su adquisición o su desarrollo en los trabajadores. 
La implementación de los modelos productivos más recientes también aparece asociada a mayores niveles de autonomía del trabajador que tienden a traducirse en una mayor responsabilidad de este sobre el proceso productivo. La importancia adquirida por la autonomía se asocia con la capacidad de los operarios para intervenir en el proceso productivo como ejecutantes y actores, y se considera que puede emerger en dos sentidos distintos: o forma parte del sistema y debe cumplirse al igual que el resto de las normas, o surge como parte de un proceso dinámico y de construcción colectiva, permitiendo la resolución de problemas. En este último caso, la autonomía asume un papel motivador y propicia el desenvolvimiento de procesos de aprendizaje (Mallet 1995), generando mayores niveles de calidad del empleo asociados con crecientes niveles de satisfacción del trabajador con las actividades realizadas. Por el contrario, cuando la autonomía remite al cumplimiento de una norma, se transforma en un nuevo elemento de evaluación del trabajador asociado con nuevas obligaciones sobre la dinámica productiva, pero sin que esto impacte positivamente en su acervo de conocimientos y capacidades y en su satisfacción personal y laboral.

Los mayores niveles de participación e involucramiento de los trabajadores también se presentan como rasgos característicos de los modelos productivos más recientes. Distintos argumentos destacan los aportes y las limitaciones de la relevancia adquirida por este factor, particularmente en lo que respecta a la calidad del empleo y la satisfacción del trabajador. Mientras que para Braverman (1978) la participación es solamente una nueva manera de garantizar el control de los trabajadores, autores como Piore y Sabel (1984) sostienen la importancia de esta dimensión para lograr esquemas y espacios de trabajo más gratificantes (Finkel 1994). Así, niveles crecientes de participación e involucramiento contribuyen a generar empleos de mayor calidad en la medida en que los trabajadores encuentran en los dispositivos ofrecidos por las empresas un medio para canalizar sus propuestas sobre mejoras del proceso productivo y estas no forman parte solamente de un propuesta discursivo para evaluar al trabajador. Cuando los canales existen pero los espacios de participación se generan a partir de la coerción y las propuestas de los trabajadores no son consideradas, este tipo de prácticas impactan negativamente sobre la calidad del empleo.

Según Zarifian (1996), existe aquello que podríamos llamar una versión "débil" de la cooperación, que reposa sobre una mejor coordinación (ordenar juntos) de los actos de trabajo. Esta versión débil es compatible con la preservación de los viejos paradigmas: se mantiene un trabajo separado y dividido, se sostiene el principio según el cual la responsabilidad no se comparte, pero se tiende a mejorar los relevos y los diálogos entre estas tareas. En el mejor de los casos, a través de esta coordinación débil, se habrán mejorado las interfases. Pero existe también una versión "fuerte" de la cooperación que plantea cuestiones más radicales. Se trata de trabajar juntos, y no solamente de coordinar trabajos separados. En las modernas condiciones de producción, esto implica comunicar, en el sentido de construir y desarrollar un espacio de intersubjetividad, de comprensión recíproca; se relaciona con establecer acuerdos sólidos acerca de objetivos, problemas, sentidos y valores de las acciones, motivaciones, etc.

En lo que respecta a la flexibilidad, mucho se ha escrito sobre su importancia para poner en práctica nuevos modelos productivos y formas de realizar el 
trabajo. Si bien es posible identificar dos tipos diferentes de flexibilidad, una pasiva y otra funcional, puede sostenerse que ambos elementos son centrales en la dinámica competitiva de las firmas en el marco de los nuevos modelos productivos. Esto es así porque la capacidad con la que cuenta la empresa para disponer de sus trabajadores (pasiva u horaria) y de las habilidades específicas de los mismos (funcional) le permite anticipar los cambios en los requerimientos de la demanda. La flexibilidad de la organización es, en parte, resultado de la flexibilidad de sus integrantes y por lo tanto, tal como lo señalan Bosch y Lehndorff (2001), la capacidad de una organización para adaptarse a condiciones y requerimientos cambiantes está íntimamente relacionada con la capacidad con la que cuentan los trabajadores para enfrentar estos mismos desafíos. El incremento de los niveles de flexibilidad impacta en las dinámicas de trabajo, en la calidad del empleo y en la calidad de vida de los trabajadores. En este caso, más que en cualquier otro, es necesario diferenciar entre la situación que enfrentan los trabajadores supeditados a un tipo u otro de flexibilidad. Mientras que la flexibilidad horaria - que afecta generalmente a personas con menores niveles de calificación - condiciona las posibilidades del trabajador de sostener sus momentos de esparcimiento y de planificar su vida extra-laboral, la flexibilidad funcional - característica de los grupos de mayor calificación relativa-implica muchas veces la extensión desmedida de la jornada laboral con una intensificación del trabajo que impacta negativamente sobre la salud del trabajador. Aunque en ambos casos es frecuente que estas dinámicas sean aceptadas por los trabajadores porque existen compensaciones mediante el reconocimiento salarial (Vidal 2013), las mismas generan un deterioro en la calidad del empleo.

Por último, se observa que el cambio en los modelos productivos ha generado en las empresas la necesidad de avanzar hacia nuevas formas de control de las actividades y de evaluación de los trabajadores. En particular, la relevancia del desarrollo de ciertas actividades intensivas en conocimiento ha puesto de relieve la incapacidad de los métodos de control típicos del modelo fordista para dar cuenta de los procesos y resultados que caracterizan a la nueva dinámica productiva. Es en este contexto que surgen lógicas indirectas de control mediante el autocontrol o la evaluación por pares, por oposición a las directas o jerárquicas. Friedman (1977) desarrolla la idea de "autonomía responsable" para definir una forma de control basada en la adaptabilidad del trabajador a ciertas iniciativas definidas por la empresa, para lo cual se requiere una supervisión mínima de los trabajadores y una elevada responsabilidad por parte de los mismos. Esta forma se contrapone al control externo o directo que se sustenta fundamentalmente en la coerción sobre el trabajador. Así, mayores niveles de calidad del empleo estarían asociados a prácticas de control vinculadas más con la primera que con la segunda de las formas consideradas.

\section{Conclusiones}

A partir de la década del setenta se han consolidado nuevas prácticas productivas asociadas a un nuevo patrón de acumulación que implicaron la pérdida de algunas de las conquistas obtenidas por los trabajadores en el marco del modo de producción anteriormente vigente. Entre ellas puede mencionarse la 
descolectivización de los procesos de negociación y la pérdida de importancia relativa de formas de contratación vinculadas con el trabajo por tiempo indeterminado, temáticas que fueron abordadas para su estudio desde las perspectivas de la informalidad y la precarización laboral.

A su vez, estas nuevas prácticas generaron grandes transformaciones en el proceso de trabajo, entre las que se destaca la relevancia adquirida por el trabajo en equipo, la participación, la polivalencia y la flexibilidad. Estos cambios han impactado sobre las condiciones laborales, pero en este caso los aportes de las perspectivas mencionadas resultaron insuficientes para captar sus implicaciones sobre la calidad de vida de los trabajadores. Es en este marco que en este trabajo se adopta la perspectiva de la calidad del empleo considerando, fundamentalmente, la dimensión de la organización productiva y la gestión del trabajo como aspectos centrales para la configuración de la misma.

Así, podemos esbozar dos conclusiones centrales. En primer lugar, se observa que los distintos factores que dan cuenta de la organización productiva y la gestión del trabajo se encuentran fuertemente relacionados entre sí. Por ejemplo, la emergencia de equipos donde los trabajadores participan en actividades operativas y de concepción se asocia con procesos de adquisición de experiencias que favorecen el desarrollo de sus capacidades, con mayores niveles de autonomía para que estos puedan intervenir en el proceso productivo, con modalidades de trabajo menos rígidas que las evidenciadas en el esquema fordista y con lógicas de control menos centralizadas y orientadas especialmente hacia el cumplimiento de objetivos.

En segundo lugar, observamos que los cambios que se sucedieron en los rasgos que caracterizan a los distintos factores que configuran la organización productiva y la gestión del trabajo, tienen un impacto discutible sobre la calidad del empleo y sobre el bienestar y desarrollo de los trabajadores. Esto surge como consecuencia de que las transformaciones productivas implementadas se orientan fundamentalmente a mejorar las condiciones de producción y competitividad de las empresas, evaluando muy raramente las implicancias de estas modificaciones sobre el principal factor productivo: la fuerza de trabajo.

Así, desde esta perspectiva, es posible pensar en mejores niveles de calidad del empleo cuando:

- El trabajo en equipo permite apropiarse de las operaciones e intervenir en las rutinas de trabajo creativamente: proponer mejoras e innovaciones, resolver problemas, optimizar procesos, etc.

- Los procesos de adquisición de capacidades se asocian tanto con una dinámica de rotación en el espacio productivo que implica el tránsito del trabajador por puestos de complejidad creciente según un esquema planificado, como con espacios de capacitación formal.

- Los niveles crecientes de autonomía colocan al trabajador en el lugar de "actor" en el proceso productivo, generan espacios para el aprendizaje y no redundan únicamente en un aumento de su responsabilidad respecto de las tareas que desarrolla.

- La dinámica de participación e involucramiento no es impuesta sino que emerge como necesidad, tanto de la empresa como del trabajador, y los aportes realizados por este último son retomados, valorados y retribuidos. 
- La organización del trabajo considera la promoción de la salud y la seguridad, garantizando los recursos que la posibilitan, al igual que la capacitación y la participación de los trabajadores en la gestión de ambientes de trabajo saludables.

- Los esquemas de flexibilidad, tanto horaria como funcional, no condicionan negativamente el desarrollo de actividades extra-laborales y la salud de los trabajadores, aún cuando exista compensación económica.

- Los mecanismos de control implementados no se sustentan en la coerción del trabajador sino que, por el contrario, implican dispositivos en los que el cumplimiento de los objetivos sea también un proceso de aprendizaje y de desarrollo de capacidades.

Algunas de estas conclusiones tienen su origen en trabajos previos en los cuales, además de la discusión conceptual y metodológica, se avanzó en el análisis empírico tomando como fuente de información encuestas a trabajadores operativos pertenecientes a sectores manufactureros específicos en Argentina. La rediscusión conceptual realizada en este artículo abre nuevos caminos para el desarrollo de futuros trabajos empíricos que incluyan a otras actividades productivas y a otros actores en el proceso de trabajo, entre ellos, las empresas. Por otra parte, consideramos relevante avanzar en el estudio de temáticas sobre las que no se ha profundizado en este trabajo, entre las cuales se encuentran los efectos de las cadenas de subcontratación y de la "deslaboralización". Si bien ambas cuestiones han sido encaradas por distintos referentes, su inclusión en relación con el abordaje presentado en este trabajo permitiría complejizar el análisis de la calidad del empleo.

\section{Referencias bibliográficas}

Aglietta, Michel. Regulación y crisis del capitalismo. La experiencia de los Estados Unidos. España: Siglo XXI Editores, 1979.

Amorín, Diego, y Analía Erbes. «Los aspectos microeconómicos de la calidad del empleo y su importancia para la estabilidad del empleo frente al ciclo económico». Ponencia presentada en $8^{\circ}$ Congreso Nacional de Especialistas de Estudios del Trabajo, (ASET), Buenos Aires, agosto de 2007.

Amorín, Diego, Andrea Pujol, Marcelo Delfini, Analía Erbes y Sonia Roitter. «Empleo de calidad: organización del trabajo y riesgos ocupacionales en empresas de la trama automotriz argentina». Estudio presentado en $12^{\circ}$ Reunión Anual de la Red PyMEs del MERCOSUR, Instituto de Economía da Universidade Estadual de Campinas, Brasil, septiembre de 2007.

Barreto, Hugo. «Concepto y dimensiones del trabajo decente: entre la protección social básica y la participación de los trabajadores en la empresa». Boletín (Cinterfor), $\mathrm{n}^{\circ}$ 151, 2001: 153-172.

Bacon, Nicolas, y John Storey. "Individualism and Collectivism and the Changing Role of Trade Unions». En Trade Unions and the New Workplace, editado por Peter Ackers, Chris Smith y Paul Smith, 2:41-76. Londres: Routledge, 1994.

Barnett, Rosalind. «Relationship of the Number and Distribution of Work Hours to Health and Quality-of-Life (QOL) Outcomes». Research in Occupational Stress and Well Being, Vol. 5, 2006: 99-138. 
Batt, Rosemary. «Who Benefits from Teams? Comparing Workers, Supervisors, and Managers». Industrial Relations, Vol. 43, $\mathrm{n}^{\circ}$ 1, 2004: 183-212.

Beatson, Mark. «Job Quality and Job Security». Labour Markets Trends, Vol. 108, $\mathrm{n}^{\circ}$ 10, 2000: 441-450.

Bosch, Gerhard, y Steffen Lehndorff. «New Forms of Employment and Working Time in the Service Economy». Final Report, TSER Programme of European Commision, Directorate General for Science, Research and Development. Gelsenkirchen: Institut Arbeit und Technik (IAT), 2001.

Boyer, Robert. La teoría de la regulación. Un análisis crítico. Valencia: Edicions Alfons El Magnànim, 1992.

Braverman, Harry. Trabajo y capital monopolista. México, D.F.: Editorial Nuestro Tiempo, 1978.

Burawoy, Michel. El consentimiento en la producción. Madrid: Ministerio de Trabajo y Seguridad Social, 1989.

Coriat, Benjamin. El taller y el robot. Ensayos sobre el fordismo y la producción de masa en la era de la electrónica. Madrid: Siglo XXI Editores, 1992a.

. Pensar al revés. Trabajo y organización en la empresa japonesa. México, D.F.: Ed. Siglo XXI, 1992b.

. Los desafíos de la competitividad. Buenos Aires: Oficina de Publicaciones del CBC-UBA, 1997.

. El taller y el cronómetro. Ensayo sobre el taylorismo, el fordismo y la producción en masa. Madrid: Siglo XXI, 2001.

Champlin, Dell. «Understanding Job Quality in an Era of Structural Change: What Can Economics Learn from Industrial Relations?». Journal of Economics Issues, Vol. XXIX, nº 3, 1995: 829-841.

De Gaulejac, Vincent. La société malade de la gestion. Idéologie gestionnaire, pouvoir managérial y harcèlement social. Paris: Editions du Seuil, 2005.

De la Garza, Enrique. «Las teorías sobre la reestructuración productiva y América Latina». En Tratado latinoamericano de sociología del trabajo, coordinado por Enrique De la Garza, 716-734. México, D.F.: Colegio de México-FLACSO-UAMFondo de Cultura Económica, 2000.

, José Gayosso y Saúl Moreno. «La Querella de las identidades ¿pasado sistemático, presente fragmentario?.» En Trabajo, identidad y acción colectiva, coordinado por Enrique De la Garza y Julio Neffa, 9-42. México, D.F.: Clacso, Universidad Autónoma Metropolitana Iztapalapa, Plaza y Valdés, S.A., 2010.

Dejours, Christophe. La banalización de la injusticia social. Buenos Aires: Topia, 2006.

Diet, Emmanuel. «Equipes pluridisciplinaires, groupalité et conflicts dáppartenance». En Agir en clinique du travail, Yves Clot y Dominique L'Huilier, 237249. Paris: Érès, 2010.

Dubar, Claude. La crisis de las identidades. La interpretación de una mutación. Barcelona: Edicions Bellaterra, 2002.

Edwards, Richard. Contested Terrain. The Transformation of the Workplace in the Twentieth Century. Londres: Heinemann, 1979.

Erbes, Analía, y Diego Amorín. «La medición de la calidad del empleo: una aproximación a partir de datos sobre tramas productivas argentinas». Estudio 
presentado en $9^{\circ}$ Congreso nacional de especialistas de estudios del trabajo, ASET, Buenos Aires, agosto de 2009.

Erbes, Analía, Sonia Roitter y Yamila Kababe. «El rol de la organización del trabajo en el desarrollo de procesos de aprendizaje». En Tópicos de la teoría evolucionista neoshumpeteriana de la innovación y el cambio tecnológico, Vol. 1, compilado por Florencia Barletta, Verónica Robert y Gabriel Yoguel, 287-317. Los Polvorines: Miño y Dávila Editores, 2014.

Ermida, Oscar. «Trabajo decente y formación profesional». Boletín técnico interamericano de formación profesional, $\mathrm{n}^{\circ}$ 151, 2001: 9-26.

Farné, Stefano. Estudio sobre la calidad del empleo en Colombia. Buenos Aires: Oficina Internacional del Trabajo, Oficina Regional para América Latina y el Caribe, 2003.

Fernández, Ernesto. «Nuevos tiempos de trabajo y calidad del empleo». En Nuevos tiempos de actividad y empleo, Rafael Muñoz de Bustillo. Madrid: Ministerio de Trabajo y Asuntos Sociales, 2003. [En línea]. http://www.academia. edu/940700/Nuevos_tiempos_de_trabajo_y_calidad_del_empleo (última consulta: 14 de diciembre de 2013).

Feldman, Silvio, y Pablo Galin. "Nota introductoria». En La precarización del empleo en la Argentina, Pablo Galín y Marta Novick, 9-17. Buenos Aires: OIT-CLACSO-Centro Editor de América Latina, 1990.

Findlay, Patricia, Arne Kalleberg y Chris Warhust. «The Challenge of Job Quality». Human Relations, Vol. 66, $\mathrm{n}^{\circ}$ 4, 2013: 441-451.

Finkel, Laura. La organización social del trabajo. Madrid: Ediciones Pirámide, 1994.

Friedman, Andrew. Industry and Labour. Class Struggle at Work and Monopoly Capitalism. Londres: The Macmillan Pres, 1977.

Gadrey, Jean. «Rapports sociaux de service: une autre regulation». Revue économique, Vol. 41, $n^{\circ}$ 1, 1990: 49-70.

Greenhalgh, Leonard, y Zehava Rosenblatt. «Job Insecurity: Toward Conceptual Clarity". Academy of Management Review, Vol. 9, $\mathrm{n}^{\circ}$ 3, 1984: 438-448.

Gittleman, Maury, y David Howell. «Changes in the Structure and Quality of Job in the United States: Effects by Race and Gender, 1973-1990». Industrial and Labor Relations Review, Vol. 48, $\mathrm{n}^{\circ}$ 3, (abril 1995): 420-440.

Grimshaw, Damian, Huw Beynon, Jill Rubery y Kevin Ward. «The Restructuring of Career Paths in Large Service Sector Organizations: 'Delayering', Upskilling and Polarisation». The Sociological Review, Vol. 50, nº 1, 2002: 89-115.

Hock, Ellen y Debra DeMeis. «Depression in Mothers of Infants: The Role of Maternal Employment». Developmental Psychology, Vol. 26, nº 2, 1990: 285-291.

Infante, Ricardo, y Máximo Vega-Centeno. «La calidad del empleo: lecciones y tareas». En La calidad del empleo. La experiencia de los países latinoamericanos y de Estados Unidos, editado por Ricardo Infante, 9-52. Santiago de Chile: Organización Internacional del Trabajo, 1999.

Lanari, María. Trabajo decente: diagnóstico y aportes para la medición del mercado laboral local. Mar del Plata: Grupo de Estudios del Trabajo de la FCEyS y la UNMDP, Ediciones Suárez, 2005.

- «Trabajo decente: significados y alcances del concepto. Indicadores propuestos para su medición». En Trabajo, ocupación y empleo. Relaciones 
laborales, territorios y grupos particulares de actividad, de Ministerio de Trabajo, Empleo y Seguridad Social, Subsecretaría de Programación Técnica y Estudios Laborales, 105-132. Buenos Aires: Ministerio de Trabajo, Empleo y Seguridad Social, 2006.

Lindenboim, Javier. «Microempresas, situación ocupacional y precariedad». En La precarización del empleo en la Argentina, Pedro Galín y Marta Novick, 107118. Buenos Aires: Ed. Centro Editor de América Latina-CIAT/OIT-CLACSO, 1990.

Linhart, Danielle. «Subjectivité collective et travail». En Agir en clinique du travail, Yves Clot y Dominique L'Huilier, 115-124. Paris: Érès, 2010.

Lipietz, Alain. «El Posfordismo y sus espacios. Las relaciones capital-trabajo en el mundo». Documento de trabajo (PIETTE-CONICET), n 4, 1994: 1-52.

Loughlin, Catherine, y Robert Murray. «Employment Status Congruence and Job Quality». Human Relations, Vol. 66, n 4, 2013: 529-553.

Mallet, Louis. «Organización formativa, coordinación y motivación». Revista europea de formación profesional, $\mathrm{n}^{\circ}$ 5, 1995: 11-17.

Méhaut, Philippe. «Transformaciones organizativas y políticas de formación: ¿Cuáles son las lógicas de las competencias?». Revista europea de formación profesional, $\mathrm{n}^{\circ}$ 1, 1994: 56-64.

Marx, Karl. El Capital. Crítica de la economía política. Moscú: Foreign Languages Publishing House, 1961 [1867].

Mocelin, Daniel. «¿Del trabajo precario al trabajo decente? La calidad del empleo como perspectiva analítica». Estudio presentado en VI Congreso Regional de las Américas, Asociación de Relaciones Laborales de la República Argentina (ARTRA), Buenos Aires, septiembre de 2008.

Neffa, Julio. Las condiciones y medio ambiente de trabajo. Presentación de la concepción dominante y una visión alternativa. Buenos Aires: CEIL PIETTE, 1995.

, Demián Panigo y Emiliano López. Contribuciones al estudio del trabajo y el empleo desde la teoría de la regulación: un marco teórico para estudiar los modos de desarrollo y su impacto sobre el empleo. Buenos Aires: CEIL PIETTE, 2010.

Novick, Marta, Ximena Mazorra y Diego Schleser. «Un nuevo esquema de políticas públicas para la reducción de la informalidad laboral». En Aportes a una nueva visión de la informalidad laboral en la Argentina, 23-42. Buenos Aires: Banco Mundial-Ministerio de Trabajo, Empleo y Seguridad Social, 2008.

Pérrilleux, Thomas. Les tensions de la flexibilité. $L$ 'épreuve du travail contemporain. París: DDB, 2001.

Piore, Michael, y Charles Sabel. The Second Industrial Divide: Possibilities of Prosperity. Nueva York: Basic Books, 1984.

Pujol, Andrea, y Guillermo Hoyos. «Organización del trabajo y acción colectiva en las trayectorias laborales de trabajadores industriales». Documento presentado en XVIII Reunión Anual Red PyMES Mercosur, Asociación Civil Red PyMes MERCOSUR-Universidad Nacional del Nordeste, Resistencia, Chaco, septiembre de 2013.

Pujol, Andrea, Sonia Roitter, Marcelo Delfini, Analía Erbes y Silvana Agüero. «Trabajar en la industria alimentaria y automotriz: una aproximación a la calidad del empleo desde la perspectiva de los trabajadores». $17^{\circ}$ Reunión Anual de la 
Red PyMEs del MERCOSUR, Universidad de San Pablo, San Pablo, octubre de 2012.

Reinecke, Gerhard, y María Valenzuela. ¿Más y mejores empleos para las mujeres? La experiencia de los países del MERCOSUR y Chile. Santiago de Chile: OIT, 2000.

Rodgers, Gerhard. The Quality of Employment: Issue for Measurement, Research and Policy. Santiago de Chile: OIT, mimeo, 1997.

y Gerhard Reinecke. «La calidad del empleo: perspectivas y ejemplos de Chile y Brasil». En Reestructuración, integración y mercado laboral. Crecimiento y calidad del empleo en economías abiertas. Santiago de Chile: OIT, 1998.

Rodríguez, Martín, Yamila Kababe, Sonia Roitter, Analía Erbes y Marcelo Delfini. "La calidad del trabajo a través del tiempo: distintos enfoques». Estudio presentado en VII Jornadas de Sociología de la UNGS, Universidad Nacional General Sarmiento, Los Polvorines, Argentina, abril de 2012.

Roitter, Sonia, Analía Erbes, Andrea Pujol, Martín Rodríguez y Marcelo Delfini. «La calidad del empleo en actividades manufactureras: un análisis de la perspectiva de los trabajadores automotrices y de la alimentación». Estudio presentado en $11^{\circ}$ Congreso Nacional de Especialistas de Estudios del Trabajo, ASET, Buenos Aires, agosto de 2013.

Rosenthal, Nicki. «More than Wages at Issue in Job Quality Debate». Monthly Labor Review, Vol. 112, $\mathrm{n}^{\circ}$ 12, (diciembre 1989): 4-8.

Sayer, Andrew. «New Developments in Manufacturing: the Just-in-time System». Capital and Class, Vol. 10, $\mathrm{n}^{\circ}$ 3, 1986: 43-60.

Slaughter, Jane. «La producción depurada y los buenos empleos». En Reestructuración, integración y mercado laboral. Crecimiento y calidad del empleo en economías abiertas. Santiago de Chile: OIT, 1998.

Smith, Adam. La riqueza de las naciones. Madrid: Alianza Editorial, 2001 [1776].

Van Bastelaer, Alois y Ralf Hussmann. «Measurement of the Quality of Employment: Introduction and Overview». Documento presentado al Joint ECE-Eurostat-ILO Seminar on Measurement of the Quality of Employment, Génova, mayo de 2000.

Verdera, Francisco. «Propuestas de redefinición de la medición del subempleo y el desempleo y de nuevos indicadores sobre la situación ocupacional en Lima». Documento de Trabajo OIT, n² 22, (septiembre 1995): 1-45.

Vidal, Matt. «Low-Autonomy Work and Bad Jobs in Postfordist Capitalism». Human Relations, Vol. 66, $\mathrm{n}^{\circ}$ 4, 2013: 587-612.

Zarifian, Phillipe. Travail et comunication. Essai sociologique sur le travail dans la grande entreprise industrielle. París: PUF, 1996. 